\title{
Characterization of the proneural gene regulatory network during mouse telencephalon development
} Julia M Gohlke ${ }^{\dagger 1}$, Olivier Armant ${ }^{\dagger 2,4}$, Frederick M Parham¹, Marjolein V Smith ${ }^{3}$, Celine Zimmer ${ }^{2}$, Diogo S Castro ${ }^{2}$, Laurent Nguyen², Joel S Parker ${ }^{3}$, Gerard Gradwohl ${ }^{4}$, Christopher J Portier*1 and François Guillemot*2

Address: ${ }^{1}$ Environmental Systems Biology Group, Laboratory of Molecular Toxicology, National Institute of Environmental Health Sciences, RTP, NC 27709, USA, ${ }^{2}$ Division of Molecular Neurobiology, National Institute for Medical Research, The Ridgeway, Mill Hill, London, UK, ${ }^{3}$ Constella Health Sciences, Durham, NC 27713, USA and "INSERM U682, Avenue Molière, 67200 Strasbourg, France

Email: Julia M Gohlke - gohlkej@niehs.nih.gov; Olivier Armant - Olivier.Armant@itg.fzk.de; Frederick M Parham - parham@niehs.nih.gov; Marjolein V Smith - msmith@constellagroup.com; Celine Zimmer - czimmer@nimr.mrc.ac.uk; Diogo S Castro - dcastro@nimr.mrc.ac.uk; Laurent Nguyen - lnguyen@ulg.ac.be; Joel S Parker - jparker@expressionanalysis.com; Gerard Gradwohl - gerard.gradwohl@titus.u-strasbg.fr; Christopher J Portier* - portier@niehs.nih.gov; François Guillemot* - fguille@nimr.mrc.ac.uk

* Corresponding authors †Equal contributors

Published: 31 March 2008

BMC Biology 2008, 6:15 doi:10.1186/1741-7007-6-15
Received: 6 August 2007

Accepted: 3I March 2008

This article is available from: http://www.biomedcentral.com/I74I-7007/6/15

(C) 2008 Gohlke et al; licensee BioMed Central Ltd.

This is an Open Access article distributed under the terms of the Creative Commons Attribution License (http://creativecommons.org/licenses/by/2.0), which permits unrestricted use, distribution, and reproduction in any medium, provided the original work is properly cited.

\begin{abstract}
Background: The proneural proteins Mash1 and Ngn2 are key cell autonomous regulators of neurogenesis in the mammalian central nervous system, yet little is known about the molecular pathways regulated by these transcription factors.

Results: Here we identify the downstream effectors of proneural genes in the telencephalon using a genomic approach to analyze the transcriptome of mice that are either lacking or overexpressing proneural genes. Novel targets of Ngn2 and/or Mashl were identified, such as members of the Notch and Wnt pathways, and proteins involved in adhesion and signal transduction. Next, we searched the non-coding sequence surrounding the predicted proneural downstream effector genes for evolutionarily conserved transcription factor binding sites associated with newly defined consensus binding sites for $\mathrm{Ngn} 2$ and Mash I. This allowed us to identify potential novel co-factors and co-regulators for proneural proteins, including Creb, Tcf/Lef, Pou-domain containing transcription factors, Sox 9 , and Mef2a. Finally, a gene regulatory network was delineated using a novel Bayesian-based algorithm that can incorporate information from diverse datasets.

Conclusion: Together, these data shed light on the molecular pathways regulated by proneural genes and demonstrate that the integration of experimentation with bioinformatics can guide both hypothesis testing and hypothesis generation.
\end{abstract}

\section{Background}

During development of the mammalian nervous system, neural progenitors within the neuroepithelium give rise sequentially to neuronal cells and glia. To achieve these well-orchestrated waves of differentiation, neuroepithelial progenitors are progressively constrained via specific 
extrinsic and intrinsic signals. By directly promoting the neuronal fate in neural progenitors, the proneural transcription factors of the bHLH family are essential regulators of neurogenesis from invertebrates to mammals [1]. Three proneural genes have been characterized to date in the embryonic mouse telencephalon: Neurogenin1 and Neurogenin2 are expressed in neuronal progenitors in dorsal telencephalon [2], which gives rise to the cerebral cortex, while Mash1 determines the fate of neuronal progenitors in the ventral telencephalon [3], giving rise to the basal ganglia. In addition to their role in the selection of neuronal progenitors within the neuroepithelium, vertebrate proneural genes have also been shown to specify neuronal subtype identities. Indeed, Ngn2 is necessary for the proper differentiation of excitatory glutamatergic projection neurons in the cerebral cortex, while Mash1 promotes the fate of GABA-ergic inhibitory interneurons in the basal ganglia [4]. Proneural transcription factors are thus critical regulators for both the initiation of neuronal differentiation and the specification of neurons into distinct regional subtypes.

In order to understand how Ngn2 and Mash1 regulate these two different aspects of neurogenesis we have developed a genomic approach incorporating the generation of microarray datasets of mice either lacking or overexpressing proneural genes, phylogenetic footprinting, and analysis using Bayesian statistical regression. The utility of Bayesian-based network analyses to determine predictive gene regulatory network structures has been demonstrated in in vitro and invertebrate models [5-7], but few attempts have been made using datasets derived from mammalian species due to the dependence of these techniques on a relatively large sample size [8]. However, this limitation may be eased through incorporation of data from other sources, such as prior information from the literature and sequence-based transcription factor binding site (TFBS) information [9].

Here, we initially identify a gene regulatory network (GRN) from a compilation of the current literature on proneural bHLH protein regulation of telencephalic development during neurogenesis. Next, microarray data from gain-of-function (GOF) analysis obtained after electroporation of either Ngn2 or Mash1 in the developing mouse dorsal or ventral telencephalon, respectively, were fused with previous and new microarray results from proneural loss-of-function (LOF) experiments [4]. This allowed us to identify novel targets of Ngn2 and Mash1, and to formally test literature-based GRN hypotheses through Bayesian statistical analysis of global gene expression patterns. A total of nine predicted proneural targets were confirmed by in situ RNA hybridization on brain slices from embryos lacking or overexpressing Mash1 or Ngn2, including several members of Notch signaling path- way (Dll1, Hes5 and Mfng) as well as the RNA binding protein Elavl4 $(\mathrm{HuC} / \mathrm{D})$. Members of the Wnt signaling pathway are predicted as targets of Ngn2 in the cerebral cortex. We then took advantage of the identification of putative downstream effectors of proneural genes to identify conserved binding sites for Mash1 and Ngn2, then scanned the sequence surrounding them for other conserved TFBSs. From this analysis, we predict Creb, Tcf/Lef, Pou-domain containing transcription factors, Sox9, and Mef $2 \mathrm{a}$ as novel co-factors (binding at a short distance) and co-regulators (binding at further distance) of proneural proteins. Finally, a novel Bayesian-based algorithm was developed to compile the microarray data, the TFBS analysis, and the literature-based network, generating a proneural GRN for the developing mouse telencephalon.

\section{Results \\ Literature-based network structure}

A GRN that describes the current understanding of proneural bHLH interactions in the developing telencephalon was developed through a review of the literature (Figure 1 ). The reader is referred Additional file 1 and several important reviews $[1,10,11]$ for more detailed descriptions of the experimental research underlying this literature-based GRN.

\section{GOF experiments}

To further identify genes regulated by the proneural factors Ngn2 and Mash1 in the embryonic telencephalon and complement existing LOF data [4], we developed a GOF approach using electroporation in a whole embryo culture. The feasibility of the GOF study was tested by injection of a Ngn2-expressing or Mash1-expressing vector in the telencephalic vesicles of E10.5 mouse embryos. The time course of Ngn2 and Mash1 overexpression was followed with Dll1 promoter-lacZ reporter transgenes monitoring Ngn2 and Mash1 activities, respectively (Figure 2(a); see also [12]). LacZ reporter activity was detected 10 $\mathrm{h}$ after electroporation of Ngn2 or Mash1 in the dorsal and ventral sides of telencephalic vesicles, respectively, and reached the highest level $18 \mathrm{~h}$ after proneural gene electroporation (Figure 2(a)). In contrast, activation by endogenous proneural proteins of the two reporters in embryos electroporated with an empty expression vector remained low. The efficiency of electroporation was similar in the control and Ngn2- and Mash1-electroporated cortices, as assessed by expression of a co-electroporated GFP plasmid before LacZ staining (Additional File 2 and data not shown). Large-scale electroporation experiments of Ngn2 in the dorsal telencephalon and Mash1 in the ventral telencephalon of E10.5 embryos were thus performed and, $18 \mathrm{~h}$ later, the electroporated tissue was processed for RNA probe preparation (see Methods). 


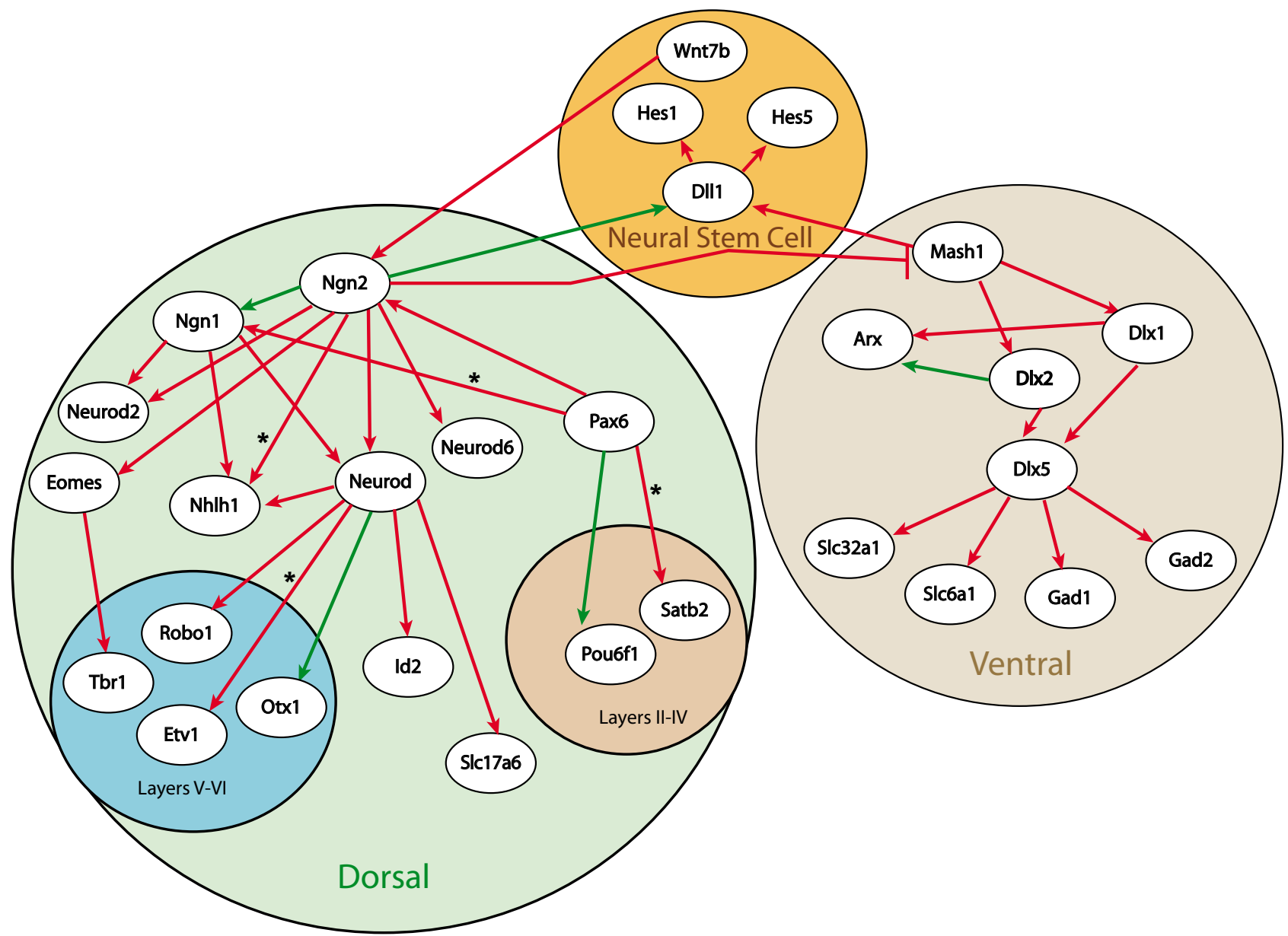

Figure I

Literature-based gene regulatory network describing proneural bHLH regulation of telencephalon neurogenesis. Activations are identified with an arrow and repressions are identified with a barred line. Those connections that are significant based on the current microarray dataset are shown in red. Significant relationships were determined through analysis of the distribution of the strength of linkage parameter ( $\beta$ ) after 500,000 MCMC simulations (Additional file 6). If more than $95 \%$ of the simulations have values above zero they are considered significant. $A *$ denotes connections that were significant, but as inhibition.

Identification of candidate Ngn2 and Mash I target genes We reasoned that by comparing the transcriptome of embryos overexpressing proneural genes with expression data obtained from current Mash1 LOF datasets and previously published Ngn2 LOF datasets [4], we would identify novel downstream effectors of Mash1 and/or Ngn2 in the developing telencephalon. For the study of Ngn2 targets, data from the cortex of Mash1-/-;Ngn2-/- double mutant mice was utilized rather than Ngn2-/- single mutants in order to avoid the compensation due to derepression of Mash1 in the cortex of the later mutants [2]. Analysis of microarray data allowed us to split potential proneural targets into three groups (Figure 2(b) and 2(c)): (i) genes regulated by both Mash1 and Ngn2; (ii) genes regulated by Mash1 only; and (iii) genes regulated by Ngn2 only. A full list of predicted targets categorized by Gene Ontology (GO) is presented in Additional file 3.

Briefly, among the 10 common targets were 3 genes involved in Notch signaling (Dll1, Hes5, and Mfng) and the RNA binding protein Elavl4 expressed in maturing neurons [13]. Specific targets of Ngn2 include genes involved in signal transduction (42 genes), transcription factors (18 genes) such as the cortical differentiation factors Nhlh1 and Bhlhb5 [14], proteins with kinase/phosphatase activity (12 genes) such as the serine/threonine 
a.

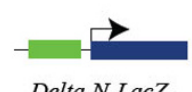

Delta N-LacZ

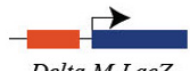

Delta $M$-LacZ

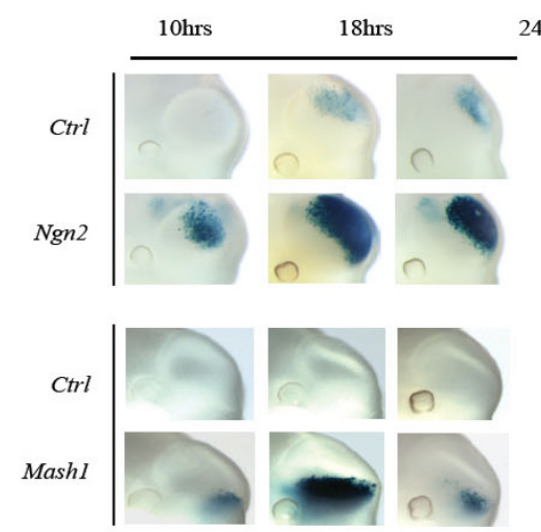

b. $\mathrm{Ngn} 2 \mathrm{GOF}$

$\square$ MashlGOF

$\square$ Mash $1-/-$

$\square$ Mash1-/-;Ngn2-/-



c.

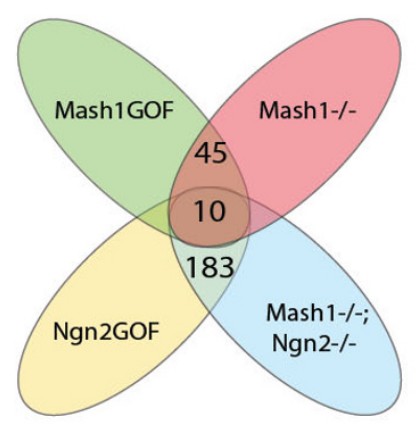

\section{Figure 2}

Mash I and Ngn2 GOF experiment. (a) Mouse embryos at I0.5 days of development were electroporated in the telencephalic vesicle with Mash I or Ngn2 expression vectors together with LacZ reporter constructs specific for either Ngn2 (0.27 kb DeltaN LacZ) or Mash I (0.16 kb DeltaM LacZ) [12,79]. The two regulatory sequences used in these reporter transgenes are located within the $0.8 \mathrm{~kb}$ distal promoter region of the DIII gene [79]. Embryos were orientated during the electroporation to target the cortex (Ngn2 vector and $0.27 \mathrm{~kb}$ DeltaN LacZ reporter) or the basal ganglia (Mashl vector and 0.16 $\mathrm{kb}$ DeltaM LacZ reporter). Ngn2 and Mash I overexpression enhances the activity of the LacZ reporter, assessed by $\beta$-galactosidase staining, compared with endogenous proneural proteins (empty expressing vector: Ctrl). Efficiency of electroporation was assessed using a GFP expression vector and was similar in all electroporated embryos (not shown). (b) Fold changes of selected potential targets of Ngn2, Mash I, or both factors in Ngn2 GOF and Mash I GOF experiments and in Mash I mutant and Mash I:Ngn2 double mutant embryos, based on normalized microarray data. A * indicates known direct targets of Mash I or Ngn2. (c) Putative targets of MashI and Ngn2 were identified through fusion of Ngn2 and Mash I LOF and GOF microarray datasets. Common targets were identified as transcripts that were decreased in Mash I LOF and Mash I;Ngn2 LOF experiments and increased in Ngn2 and Mash I GOF experiments. Mash I targets are those transcripts that were only decreased in Mash I LOF and increased in Mash/ GOF. Ngn2 targets are those transcripts that were only decreased in Ngn2/MashI LOF and increased in Ngn2 GOF. A cut-off of I.3-fold was used as described in more detail in Methods. A full list of predicted targets categorized by GO is available in Additional file 3. 
kinase $D c x$, already shown to be directly regulated by Ngn2 in the cortex [15] and other cortical genes such as the cell adhesion molecule Ephrin A4 [16]. Interestingly, several components of the Wnt pathway are predicted targets of Ngn2 including Wnt7b [17], DIX domain containing $1[18], F z d 1, F z d 3$, and Tax1bp3. It is noteworthy that NeuroD, a gene considered as directly regulated by Ngn proteins [19], was decreased in the Ngn2-/- embryos, but was not significantly upregulated after Ngn2 electroporation in microarray experiments, suggesting that the window for analysis of electroporated embryos was not suitable. Indeed, in situ hybridization analysis showed that electroporation of Ngn2 does not induce NeuroD expression in the telencephalon at $24 \mathrm{~h}$, but only at $48 \mathrm{~h}$ after electroporation (Additional file 2). A similar delay has been reported for induction of NeuroD by the related gene Ngn3 in the intestine [20]. This suggests either that NeuroD is not a direct target of Ngn2, or that its expression also requires another factor that is not present at the time of electroporation. Involvement of other genes in the regulation of NeuroD is supported by the lack of a dramatic change in NeuroD expression in Ngn2 null mutant telencephalon [21].

Finally, putative targets of Mash1 include transcription factors (seven genes) such as the LIM homeobox transcription factor Isl1, which is potentially involved in the specification of GABAergic projection neurons in the striatum [22], and factors involved in signal transduction such as the GABA vesicular transporter Slc32a1/VIAAT [23] and the gene GP38/Podoplanin [24], the Notch regulated protein Nrarp shown to destabilize the Notch intracellular domain (NICD) [25-27], and Lfng, a modulator of Notch signaling regulated directly by Mash1 in the telencephalon [12]. These putative target genes form the basis for subsequent bioinformatics and network analyses to predict novel co-factors, co-regulators, and GRN connectivity.

\section{Validation of predicted target genes}

To confirm the microarray data, several of the predicted novel targets of Ngn2 and Mash1 were validated through RNA in situ hybridization analysis of wild type and mutant embryos (Ngn2-/- and Mash1-/-), and of embryos overexpressing Ngn2 or Mash1 (Figure 3 and Additional file 4). The predicted Ngn2 targets Nhlh1, Mfng, and Elavl4 are mostly expressed at E13.5 in the mantle zone of the dorsal telencephalon, while Rbdh4 and $z f p 238$ are also expressed in the ventricular zone. All five genes are downregulated in the medial part of the dorsal telencephalon of $\mathrm{Ngn} 2$ mutant embryos, indicating that $\mathrm{Ngn} 2$ is indeed required for normal expression of these genes (Figure 3(a)). We also show that Ngn2 can induce expression of the same predicted targets when overexpressed by electroporation in the telencephalon of E10.5 embryos. At this stage, their expression is barely detectable in neurons that begin to accumulate above the ventricular zone of the dorsal telencephalon (left telencephalic vesicles in Figure 3(a)). Expression of Nhlh1, Mfng, zfp238, Rbdh4, and Elavl4 is strongly upregulated after electroporation of a Ngn2-expressing vector in the dorsal telencephalon (right telencephalic vesicles in Figure 3(a), marked by ${ }^{*}$ ). Thus, both LOF and GOF analysis confirm that five predicted targets are indeed regulated by Ngn 2 in the developing mouse telencephalon.

The predicted Mash 1 targets Mfng and Pdpn are predominantly expressed in the ventricular zone of the ventral telencephalon at E12.5, while Gadd45g is predominantly expressed in the subventricular zone and Elavl4 in the mantle zone (Mnfg and Elavl4 are common candidate targets of Mash1 and Ngn2). All four genes are downregulated in the telencephalon of Mash1 mutant embryos particularly in the medial part of the ventral telencephalon, which is most severely affected in Mash1 mutant embryos [3] (Figure 3(b)). Ectopic expression of Mash1 in the dorsal telencephalon of E10.5 embryos strongly induces expression of the four genes (Figure 3(b)). Mash 1 also rapidly induces expression of Gadd45g and Mfng in the embryonal carcinoma cell line P19, as detected by quantitative reverse transcription polymerase chain reaction (RT-PCR; see Additional file 5). These data suggest that many of the genes identified by microarray analysis of Mash1 and Ngn2 GOF and LOF experiments are indeed regulated by Mash1 and Ngn2.

\section{Bayesian analysis of literature-based GRN}

Connectivity in the literature-based network structure was quantified based on the GOF and LOF microarray gene expression datasets. This Bayesian-based method removes the need to rely on fold change cut-offs by examining the strength of the predicted relationships based on concurrently evaluating variability in gene expression patterns of several genes of interest across control and perturbation experiments. Significant connections based on this analysis (the fifth percentile of the posterior density for $\beta$ is greater than zero [8]) are highlighted in red in Figure 1. Our analysis predicts $86 \%(31 / 36)$ of the connections as significant based on the microarray dataset; however, four are considered inhibitory interactions instead of activations, suggesting possible feedback loops between Pax6 and Ngn1, between Satb2, Ngn2 and Nhlh1, as well as between NeuroD and Etv1 (Additional file 6). Specificity of our method was estimated through the application of 100 randomly generated datasets by permutation of gene labels using the entire microarray dataset. This analysis results in an estimated false positive rate of $8.2 \%$, therefore the chances of seeing 31 significant connections out of 36 is very low $(p<0.001)$. Two of the connections originating from Ngn2 (to Ngn1 and Dll1) are not considered 

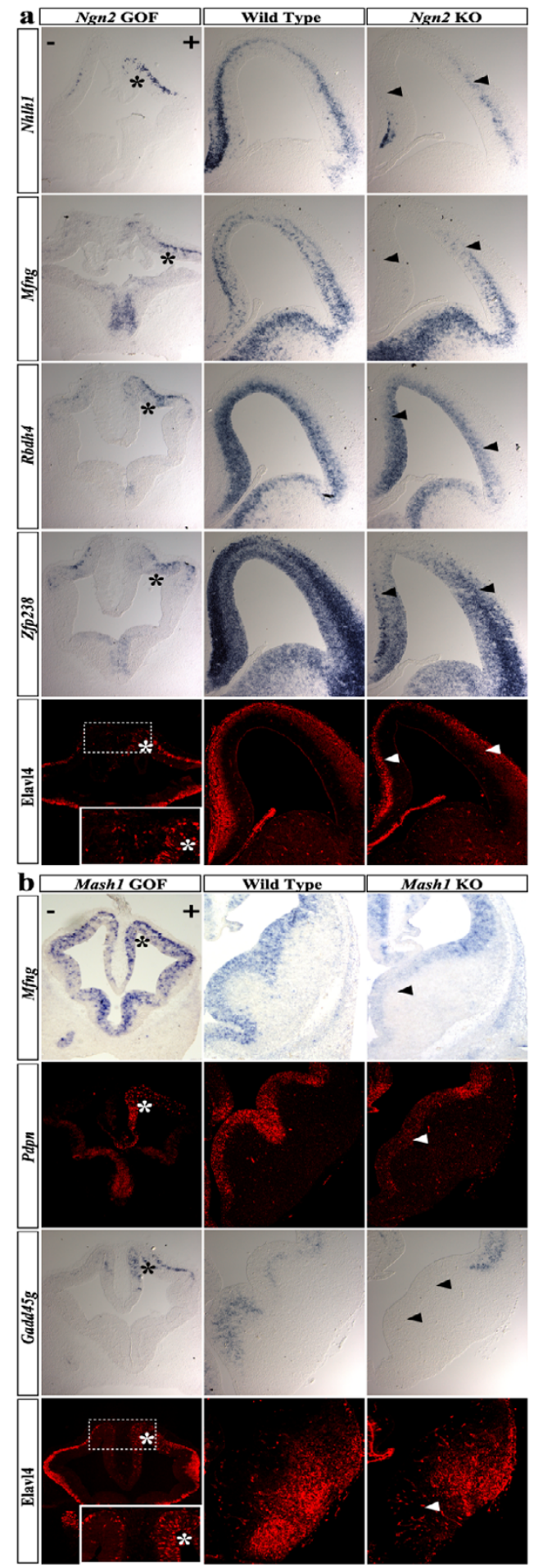

Figure 3

Expression analysis of predicted Ngn2 and Mash I targets through in situ hybridization of overexpressing and mutant embryos. (a) Expression of the predicted Ngn2 targets Nhlh I, zfp238, Rbdh4, Mfng, and Elavl4 (the latter two are predicted common targets of Ngn2 and Mash I) in EI0.5 embryos electroporated with a Ngn2 expression vector and cultivated for one day (left panels), in EI 3.5 wild-type embryos (middle panels), and in EI3.5 Ngn2 mutant embryos (right panels). (b) Expression of the predicted Mash I targets Gadd45g, Pdpn, Mfng, and Elavl4 in EI0.5 embryos electroporated with a Mash I expression vector and cultivated for one day (left panels), in EI2.5 wild-type embryos (middle panels), and in EI2.5 Mash I mutant embryos (right panels). A* in the left panels indicates cells overexpressing the target genes. Arrowheads in the right panels indicate cells having downregulated the target genes. 
significant based on the current analysis. As the algorithm relies on linear relationships between genes, its inability to detect these two connections may be a result of the non-linear variability between $\mathrm{Ngn} 2$ and its targets that is created by the large increase in Ngn2 transcript levels after electroporation. In addition, the compensatory action of Mash1 in the dorsal telencephalon of the Ngn2-/- mutants further complicates the algorithm's ability to detect connectivity to common targets such as Dll1.

\section{Bioinformatics prediction of Ngn2 and Mash I co-factors}

The identification of potential downstream effectors of Mash1 and Ngn2 allowed us to examine the occurrence of defined TFBSs in the conserved sequence surrounding those genes. The E-box recognized by bHLH proteins is a degenerate 6 bp (base pair) motif (CANNTG). However, recent evidence suggests that Mash1 and Ngn2 have different preferences for the two central residues and that their consensus binding sequences extend even outside of the E-Box motif (see $[12,28,29]$ and FG and DC, unpublished data). Based on these and other data, we defined different consensus binding sequences for Mash1 (GCAGSTGK or CAGSTG) and Ngn2 (CANTWG) (Additional file 7).

To identify potential co-factors for Ngn2 or Mash1, we employed phylogenetic footprinting and TFBS search algorithms on a subset of the predicted targets based on the microarray dataset (Figure 4(a)). First, 58 conserved (human, mouse, chicken, frog, and fish) putative Ngn2 binding sites surrounding 11 of the predicted Ngn2 target genes and 56 conserved putative Mash 1 binding sites surrounding 14 of the predicted Mash1 target genes and 6 of the common target genes were identified (see Methods for further details). Subsequently, we identified TFBS from the TRANSFAC library co-occurring specifically with the putative Ngn2 binding sites in Ngn2 targets or with the putative Mash1 binding sites in Mash1 and Mash1/Ngn2 common target genes using Fisher's exact two-sided test for significance (Figure 4(b)). No specific co-factors for Mash1 were identified in this analysis, whereas CAMP response element (CRE; bound by CRE binding protein (Creb)), Yy1, and Nkx binding sites were significantly enriched near Ngn2 binding sites when compared with the sequence around Mash1 binding sites (Figure 4(b)). Several of the predicted co-factors have dorsal/ventral restricted expression patterns in the embryonic telencephalon, consistent with a role in neuronal specification (Figure 4(c)).

\section{Bioinformatics prediction of Ngn2 and Mash I co- regulators}

Next we sought to predict potential co-regulators that may bind at regulatory modules independent from those that bind Ngn2 or Mash1. In particular, we were interested in identifying transcription factors that may confer regula- tion at modules closer to the TSS (transcription start site). In order to efficiently search for co-regulators, we identified evolutionary conserved TFBS (between human and mouse) using the CONFAC algorithm [30] to analyze a shorter sequence $(10,000 \mathrm{bp})$ in the $5^{\prime}$ flanking region of 31 predicted Mash1, Ngn2, and common targets (Additional file 8 ). To predict putative common co-regulators, we tested for enrichment of the identified TFBS in Ngn2 target genes and Mash1/common target genes compared with a set of 250 randomly selected genes using the MannWhitney statistical test. We identified 41 potential Ngn2and Mash1-specific co-regulators by testing for TFBS enrichment in Ngn2 targets versus Mash1 targets and vice versa. We identified 14 putative co-regulators from these analyses after eliminating those transcription factors that are not expressed in either the dorsal or ventral developing telencephalon based on microarray transcript levels (Additional file 9). Predicted common co-regulators include E2f1, Tef, Nfy, Egr1, Hes1, and Pou-domain containing factors. Predicted co-regulators were specifically enriched in Ngn 2 targets when compared with Mash1 and common targets include Sox9, Creb, Tcf4, Lef1, Mef2a, and Yy1. No transcription factors were significantly enriched when Mash1 target genes were compared against Ngn2 target genes. This approach identified all five of the predicted Ngn2 co-factors found in the previous analysis, as well as identifying several transcription factors that may co-regulate the putative Ngn2 and Mash1 targets at independent regulatory modules including E2f1, Egr1, Hes1, and Nfy in both Ngn2 and Mash1 targets and Sox9 and Mef2a in Ngn2 target genes.

Through interrogation of online databases of in situ hybridization in serial brain sections across development $[31,32]$, we confirmed the expression of several predicted co-factors/co-regulators in the developing dorsal and ventral telencephalon (Additional file 10). Interestingly, several of the predicted co-factors/co-regulators, including Sox9, Crebbp, Creb1, Tcf4, Lef1, Pou6f1, Pou2f1, Pou3f1, Tef, Hes1, and E2f1, show appreciable expression in the ventricular zone of dorsal and/or ventral telencephalon, where proneural bHLH proteins are expressed. Furthermore, direct protein-protein interactions between proneural bHLH proteins and Crebbp, Tcf4, and Mef2a are reported in the human protein reference database [33].

\section{Bayesian network analysis with an informative prior structure}

To provide an integrated view of the network regulated by proneural genes, information obtained from the literature, novel expression data, and phylogenetic footprinting analyses were quantitatively linked through application of the Bayesian-based TAO-Gen algorithm [34] with the addition of an informative prior structure (Figure 5, Additional file 11). Nodes represented in the network include 
a.

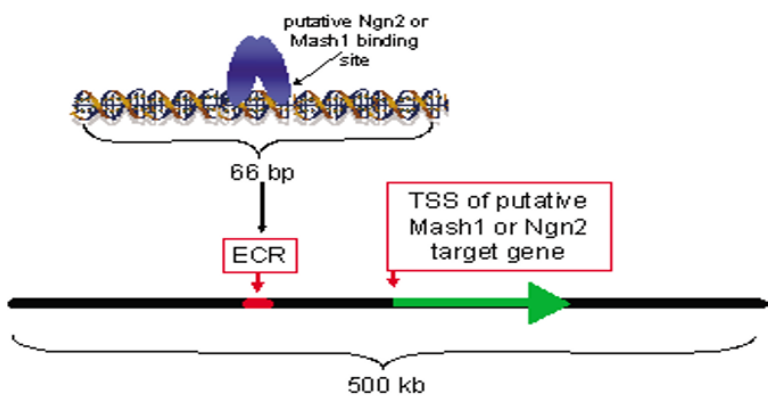

b.



c.

\begin{tabular}{|ccc|}
\hline Gene & $\begin{array}{c}\text { Dorsal vs. Ventral } \\
\text { Genession (fold change) }\end{array}$ & $\begin{array}{c}\mathbf{1 . 5} \text { Fold } \\
\text { Cutoff }\end{array}$ \\
\hline Creb & 3.13 & dorsal \\
Crebbp & 10.02 & dorsal \\
Tcf4 & 2.04 & dorsal \\
Lef1 & 2.97 & dorsal \\
Pou3f3 & 1.66 & dorsal \\
Yy1 & 0.49 & ventral \\
Pou3f4 & 0.44 & ventral \\
Pou3f1 & 0.33 & ventral \\
Pou3f2 & 0.94 & common \\
Pou6f1 & 0.66 & common \\
Pou2f1 & 0.76 & common \\
\hline
\end{tabular}

\section{Figure 4}

Identification of potential co-factors for Ngn2 and Mash I using bioinformatics approaches in comparative genomics. (a) Putative Ngn2 and Mash I bindings sites were identified in ECRs in a $500 \mathrm{~kb}$ region surrounding $34 \mathrm{Ngn} 2$ and Mash I target genes (list of target genes analyzed in Additional file 8). Other TFBSs were identified within 30 bp of the putative Ngn2 or Mashl binding site. (b) TRANSFAC TFBSs that co-occurred with Ngn2 binding sites versus Mash I binding sites surrounding Ngn2 target genes versus Mash I and common target genes, respectively. A total of $58 \mathrm{Ngn} 2$ sites and 56 Mash I binding sites were analyzed. A* denotes significantly enriched TFBSs in the sequence surrounding Ngn2 sites versus Mash 1 sites (Fisher's exact two-sided test with $p \leq 0.05$ ). (c) Comparison of microarray gene expression values of potential Ngn2 and Mash I co-factors in wild-type dorsal and ventral telencephalon tissues where column three presents results using a I.5-fold change cut-off value for categorizing gene expression as either preferentially dorsal, ventral, or both (common). 
several candidate Mash1 and Ngn2 target genes from the fusion and sorting of the GOF/LOF microarray datasets, as well as candidate co-factors and co-regulators from the phylogenetic footprinting analyses. An informative prior structure considered all significant literature-based connections as required (diagrammed using thicker lines) and used the TFBS information from the phylogenetic footprinting analyses to weight connections in which TFBS information was found. For example, a conserved Sox9 TFBS was identified in the sequence surrounding Lfng, therefore a preference is given to Sox9 being a parent to $L f n g$, which results in a slightly higher probability that a linear relationship between these two genes will be significant in the posterior distribution. We have confirmed the reliability of the TAO-Gen algorithm as well as the utility of the informative prior structure through direct comparisons with results obtained from another Bayesian network algorithm [35,36], as well as with results obtained using TAO-Gen without an informative prior (Additional file 1).

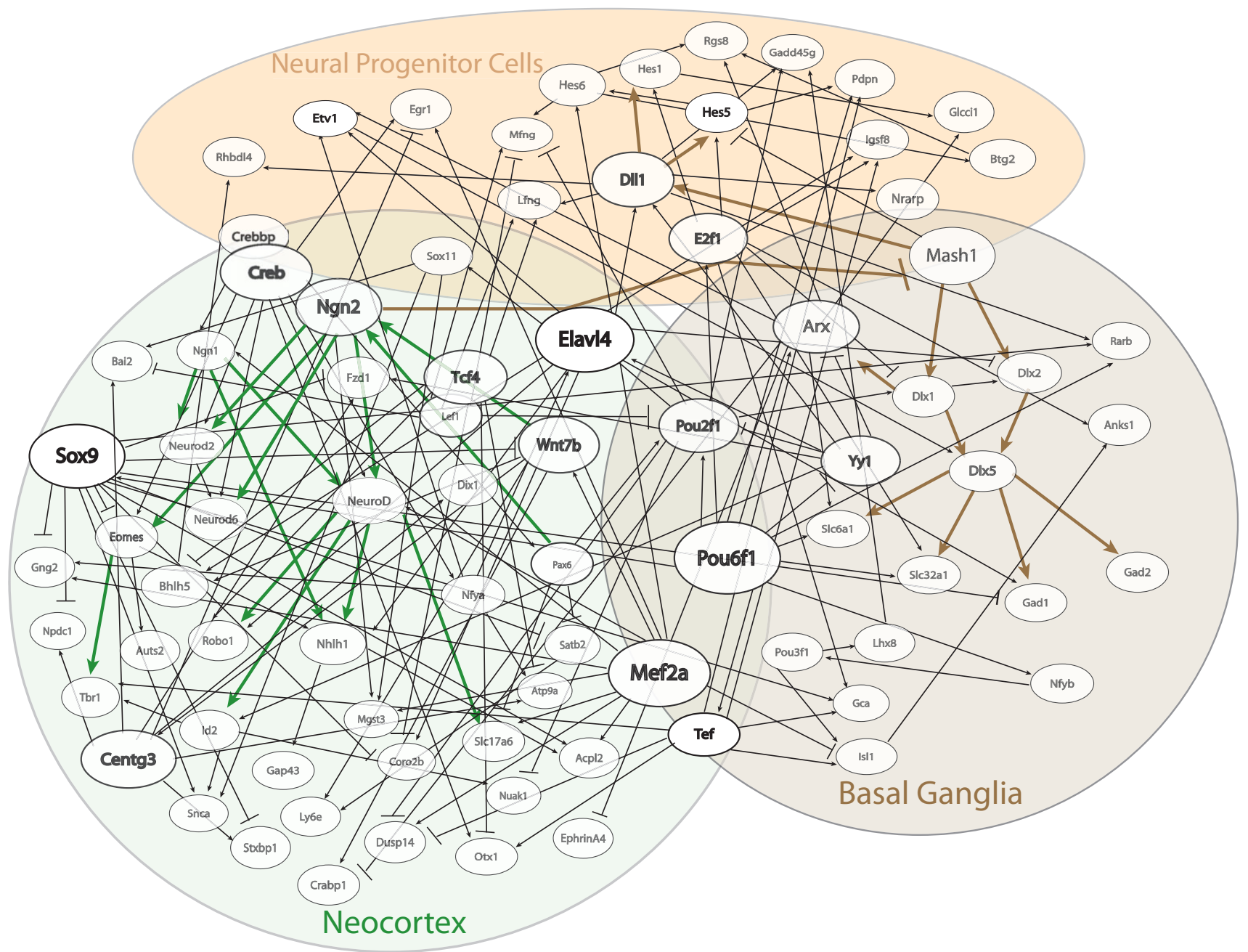

Figure 5

Algorithm-based gene regulatory network structure for dorsal and ventral telencephalon development. The Bayesian-based TAO-Gen algorithm was implemented with an informative prior structure to predict the optimal network structure based on the LOF and GOF microarray datasets, evolutionarily conserved TFBS data, prior literature-based knowledge, and spatial and time-specific expression patterns. To highlight the key regulators, the nodes representing genes predicted to be the parent of at least nine other genes are largest in size (Sox9, Mef2a, Elavl4 and Pou6fl), whereas those that are predicted to regulate at least five other genes are medium in size (Ngn2, Centg3, Tef, Tcf4, Wnt7b, Pou2fI, Yyl, DIII, E2fI, Arx, and $\mathrm{Creb}$ ). See Additional file II for matrix of connectivity and Methods for a more detailed description of algorithm. 
The resulting Bayesian-based network structure predicts 174 linkages ( 27 of which are significant lit-based connections) between the set of 82 genes. Sox9, Mef2a, Elavl4, and Pou6f1 are predicted as the most prolific co-regulators of the target genes, with 14, 12, 9, and 12 children, respectively. Furthermore, our analysis predicts Creb1, Crebbp, and Yy1 as the most likely candidates for dorsally expressed Ngn2 co-factors, and supports a synergistic interaction between Pou-domain containing transcription factors and bHLH proneural proteins in the regulation of common target genes, which is consistent with previous studies [12]. We also identified several transcription factors as candidate co-regulators of Ngn2 target genes, including Hes1, Egr1, Nfy, Mef2a, Tef, and Sox9, whereas Pou6f1 is a predicted co-regulator of Mash1 target genes. Interestingly, these different transcription factors have been previously implicated in regulatory processes in other developmental contexts [37-42], consistent with a potential role in regulating neurogenesis during telencephalon development.

\section{Discussion}

As the amount of experimental data grows and becomes more complex, the power of computational models to identify mechanisms of biological processes by integrating diverse sets of experimental data is being realized, particularly in organisms where high throughput perturbation analyses have been developed, including Saccharomyces cerevisiae, Caenorhabditis elegans, and Strongylocentrotus purpuratus [43-47]. However, finding and quantifying mammalian networks has been challenging due to the data requirements of current methods. In the present analysis, we have demonstrated the utility of bioinformatics approaches for elucidation of a GRN describing proneural bHLH (Ngn2 and Mash1) transcription factor regulation of murine telencephalon specification.

To develop a robust GRN, we first measured global gene expression patterns in GOF and LOF analyses to identify novel putative target genes for Ngn2 and Mash1, several of which were validated by in situ hybridization in slice preparations from mutant and electroporated mouse embryos. Using Bayesian network analysis, we have corroborated 25 literature-based GRN hypotheses by quantifying connections based on the compiled microarray datasets.

As highly conserved long-range enhancers are known to be particularly important for developmentally regulated genes [48-50], we utilized phylogenetic footprinting analyses to identify putative long-range enhancers in several predicted Ngn2 and Mash1 target genes. This approach suggests that no putative co-factor binding sites are specifically associated with conserved Mash1 binding sites in both Mash1 and common target genes, consistent with an instructive and relatively context-independent role for
Mash1 in ventral cell fate determination [51]. In addition, the analysis identifies several conserved TFBSs in close proximity to Ngn2 binding sites surrounding the Ngn2 target genes, consistent with previous findings supporting a more cell context-dependent role for Ngn2 in dorsal telencephalic fate specification [51]. In addition, we hypothesized that important regulatory modules that bind transcription factors other than Ngn2 and Mash1 may be present surrounding the identified target genes. Therefore, our second comparative genomics analysis was designed to search for conserved TFBS enriched in target genes, but not necessarily in close proximity to Mash 1 or Ngn 2 binding sites. Using a novel Bayesian network analysis approach with an informative prior structure, we were able to synthesize the knowledge gained from each of the above experimental and computational approaches to predict connectivity between the novel target genes, cofactors, and co-regulators.

Our resultant GRN predicts that Creb1 and Crebbp are the most likely candidates for a dorsally expressed Ngn2 cofactor regulating cortical targets such as Neurod6, Eomes and NeuroD2. Interestingly, previous analyses have indicated interactions between bHLH transcription factors, Creb, and the Creb binding protein (CBP/P300 complex) in the differentiation of several cell types [52,53], as well as in neurotrophin-mediated expression of $v g f$ [54]. Most notably, a conserved Neurod6 promoter has recently been shown to contain both Creb and E-box binding sites and is activated via cAMP exposure [55]. Yy1 is also a potential Ngn2 co-factor, yet our microarray data suggest that $Y y 1$ mRNA expression is confined to the ventral telencephalon. This finding suggests an inhibitory role for Yy 1 in Ngn2-regulated transcription, which is supported by evidence showing Yy1 inhibition of both BMP induced cell differentiation [56] and Notch transcriptional activity [57].

Our GRN also supports a synergistic interaction between Pou-domain containing transcription factors and bHLH proneural proteins in the activation of several target genes. Interestingly, both Pou6f1 and Mash1 have been identified as important mediators of oligodendrocyte development $[58,59]$, and Pou6f1 is thought to synergistically interact with members of the Sox family $[60,61]$. Moreover, other members of the Pou domain, class 3 family of transcription factors, Brn1 or Brn2, which exhibit similar affinity with Pou6f1 for certain binding sites $[62,63]$ have been experimentally shown to co-regulate several Mash1 targets in the ventral telencephalon [12].

Finally, our analysis predicts connectivity between Sox9, $F z d 1$, and $W n t 7 b$, which is consistent with a recent report suggesting Sox9 regulation through Wnt signaling [64]. Sox9 may serve as an inhibitory factor for several Ngn2 
target genes including Gng2, Npdc1, Eomes, Fzd1, and Coro $2 b$, consistent with the opposing roles of Sox9 and $\mathrm{Ngn} 2$ in the specification of glial fate $[35,36]$. We also show that Mef2a, which has been shown previously to have complex regulatory functions in neuronal differentiation and plasticity [38-40], may co-activate several of the predicted Ngn2 targets, such as Neurod, Ngn1, Wnt7b, Mgst3, Gng2, Acpl2, and Dusp14. In addition, our findings suggest that transcriptional regulators downstream of Wnt signaling (Tcf4/Lef1) may bind to regulatory modules that also bind Ngn2, which is consistent with the role of Wnt signaling in the specification of the dorsal forebrain [6567l, and offers a hypothesis in which coordinated Wnt activation and Ngn2 expression act in concert to transcriptionally activate target genes. Interestingly, a recent report suggests that Wnt pathway-initiated neural differentiation, but not proliferation, requires specific interactions between Tcf/ $\beta$-catenin and Crebbp [68]. Another intriguing aspect of our analysis is the prediction of Elavl4 as an important regulator of Ngn2 targets, including Bhlhb5, Robo1, Nhlh1, Coro2b, Sox11, and Dll1, possibly through stabilization of mRNA [69]. It is of note that two related mRNA stabilization genes, Elavl2 and Elavl3, are also predicted targets of Ngn2.

Our current research identifies important research steps for further refinement of the GRN. The cyclic nature of delayed negative feedback in the Notch pathway is thought to act as a molecular clock regulating the timing of several developmental processes [70]. Future production of robust time-series datasets will allow for application of Bayesian methods that are not limited to the discovery of acyclic networks and linear relationships [71]. Several recent reports have suggested connectivity between Notch and Wnt pathways [27,68,72,73]. For example, one way in which the Notch pathway may regulate Wnt signaling is through Lef protein stabilization by Nrarp activation [27]. However, because no data currently exist that would allow quantitative prediction via direct protein-protein interactions, we are unable to predict this relationship in the current network analysis. Nonetheless, our network analyses of transcriptional regulation offers other hypotheses such as a Tcf/Lef regulation of $L f n g$ and $\mathrm{Mfng}$, which is consistent with evidence during somitogenesis suggesting a Wnt mediated regulation of $L f n g$ expression [74].

One important application of the network analysis is the prediction of the most useful perturbation or chromatin immunoprecipitation experiments for resolving the overall network structure [75]. This is particularly relevant to studies in mammalian species, in which perturbation analyses are much more time and resource intensive. Our network analysis would suggest Centg3 and Elavl4 are important candidates for perturbation and subsequent global gene expression analysis for further network resolution, as they are both highly connected nodes and little is known about their function in the developing telencephalon. Interestingly, Centg3 may protect against neurodegeneration in Polyglutamine diseases [76]. The central role of Elavl4, which regulates through mRNA stabilization [69], highlights the importance of moving beyond cis regulatory binding to elucidate network relationships.

\section{Conclusion}

In this manuscript, we have combined a number of different approaches to identify regulatory relationships that are important for mammalian telencephalic development, using existing knowledge from the literature, global gene expression data from GOF and LOF studies, bioinformatics-based sequence analyses, and Bayesian network algorithms. Systems biology methodologies that take into account several data sources, such as that presented here, will aid in more rapid identification and quantification of gene regulation which is useful for discovering critical steps in the progression of normal and perturbed human telencephalon development. Eventually, the linkage of mammalian genetic perturbation network analysis with protein interactions and ultimately phenotypic outcome will become possible, as has been initiated in invertebrate models. The methods developed and applied in this manuscript are a first step towards this broader goal.

\section{Methods}

\section{Electroporation of mouse embryos}

Embryos obtained from CBA/CA X C57Bl/10 crosses were dissected without removing placental membranes at embryonic day E10.5, and were transferred into Tyrodes solution [77]. At this stage Mash1 and Ngn2 are already expressed in the presumptive basal ganglia and cortex, respectively. The neuroepithelium is thus competent for proneural activity and GOF studies should allow the detection of genes regulated by Mash1 or Ngn2. Embryos were precultured for $2 \mathrm{~h}$ into a 'precision incubator' (BTC Engineering, Milton, Cambridge, UK) at $37^{\circ} \mathrm{C}$ with $65 \%$ oxygen in $75 \% \mathrm{v} / \mathrm{v}$ Rat serum $+25 \% \mathrm{v} / \mathrm{v}$ Tyrodes solution and $2 \mathrm{mg} / \mathrm{ml}$ of glucose. Both telencephalic vesicles were injected for microarray experiments and with only one vesicle for embryos processed for in situ hybridization using a FemtoJet Microinjector (Eppendorf) with $2 \mu \mathrm{l}$ of a solution containing $3 \mu \mathrm{g} / \mu \mathrm{l}$ Mash1- or Ngn2-pCAGGS expression vector [78] and $2 \mu \mathrm{g} / \mu \mathrm{l}$ GFP control vector. Electroporation was performed in Tyrodes solution in a CUY520P20 chamber (Nepagene, Japan) using a BTX Electro Square Porator (Eppendorf), with the following settings: $70 \mathrm{~V}$, five pulses, $50 \mathrm{~ms}$ at $1 \mathrm{~s}$ intervals. Electroporated embryos were cultivated in Rat serum supplemented with glucose as above, for the indicated time. After $18 \mathrm{~h}$, heart-beating embryos were dissected under a UV binocular microscope and the electroporated (GFP- 
expressing) tissue was dissected under the microscope and homogenized immediately in $300 \mu \mathrm{l}$ Trizol (Invitrogen). The choice of the time of collection of the tissue was based on maximal expression of the Dll1 promoter-lacZ reporter located in the $0.8 \mathrm{~kb}$ distal promoter region described previously [79] (see Figure 2(a)) and on a more detailed analysis by quantitative RT-PCR of the time course of induction of Dll1 by Mash1 in P19 cells [12]. This work showed that induction of this direct target of Mash1 becomes detectable 4-7 h after Mash1 expression but reaches a plateau only after about $15 \mathrm{~h}$. Therefore, this time of collection maximizes the detection of putative direct targets; however, it does not rule out the possibility of detecting indirect targets as well. Total RNA was extracted following manufacturer recommendations and resuspended in $12 \mu \mathrm{l}$ of diethylpyrocarbonate (DEPC)treated water (Ambion). Between 3 and 10 electroporated cortices or basal ganglia were pooled to produce a minimum of $1 \mu \mathrm{g}$ total RNA. The preparation of probes and hybridization to MG430 2.0 chips were performed following Affymetrix guidelines.

\section{RNA in situ hybridization and immunocytochemistry}

Electroporated embryos were washed for $30 \mathrm{~min}$ at $4{ }^{\circ} \mathrm{C}$ in phosphate buffered saline (PBS) $1 \times$, fixed in PFA $4 \%$ for 3 $\mathrm{h}$, washed again in PBS $1 \times$ and incubated in $15 \%$ sucrose phosphate buffer $0.12 \mathrm{M}(\mathrm{PB}), \mathrm{pH} 7.2$, overnight at $4{ }^{\circ} \mathrm{C}$, incubated in gelatin $7.5 \%$ sucrose $15 \% \mathrm{~PB}$ at $42^{\circ} \mathrm{C}$ and frozen in isopentane at $-40^{\circ} \mathrm{C}$. Wild-type and mutant embryos (Ngn2-/- and Mash1-/-) at stage E12.5 or E13.5 were fixed overnight in $4 \%$ paraformaldehyde (PAF) at $4{ }^{\circ} \mathrm{C}$, incubated overnight as before. Embryonic sections were performed at $10 \mu \mathrm{M}$ using a Microm cryostat. In situ hybridizations were carried out as described previously [12], with NBT/BCIP or fluorescent substrate in the case of Gp38/Podoplanin [80]. Mouse Elavl4 (HUC/D) polyclonal antibody was used as described previously [12]. All of the in situ analyses performed are summarized in Additional file 4. Briefly, eight genes putatively regulated by Ngn2 and eight genes regulated by Mash1 were selected based on their levels of downregulation and upregulation in the microarray data, as well as their potential involvement in neuronal development $(\mathrm{Mnfg}, \mathrm{Lnfg}, \mathrm{Lhx} 8, \mathrm{HuC/D}, \mathrm{Nscl1})$ or expression in the developing nervous system (Gp38/ podoplanin, Rhomboid, Nrarp). Two out of eight Ngn2 candidate genes were not detected in situ in the cortex of WT embryos and were not tested further. Candidates showing a consistent regulation in Ngn2 or Mash1 LOF mutant embryos (five out of six expressed genes for Ngn2 and six out of eight genes for Mash1) were further analyzed by in situ hybridization on electroporated GOF embryos.

\section{PI9 cell transfection and quantitative RT-PCR}

P19 embryonal carcinoma cells are pluripotent cells that specifically differentiate into neurons when induced by retinoic acid or when transfected with Mash1, Ngn1, or NeuroD expressing vectors [81,82]. We seeded 250,000 P19 cells into $21 \mathrm{~cm}$ culture dishes in DMEM (Gibco) supplemented with $5 \%$ goat serum and incubated overnight at $37^{\circ} \mathrm{C}$. Cells were transfected in duplicate with $2 \mu \mathrm{g}$ Ngn2-, Mash1-, or empty pCAGGS vectors and $0.1 \mu \mathrm{g}$ GFP control vector mixed with Lipofectamine 2000 (Invitrogen) following the manufacturer's recommendations. Total RNA extraction was performed in $2 \mathrm{ml}$ Trizol (Invitrogen). RNA pellets were resuspended in $50 \mu \mathrm{l}$ DEPC treated water (Ambion) and the RNA concentration was determined by spectrophotometry. A total of $2 \mu \mathrm{g}$ RNA was treated with 10 units DNase I (Invitrogen) and reverse transcribed with Superscript III (Invitrogen). Quantitative PCR was performed in duplicates with SYBR Green (Roche) on a Light cycler apparatus (Roche). A cDNA from hydroxymethylbilane synthase was used as a reference for normalization. Primer sequences are available from the authors upon request.

\section{Global gene expression analyses}

Twenty-eight separate gene expression datasets were used for the identification and quantification of GRNs for forebrain development using either the U74A and U74B or MOE430 2.0 Affymetrix microarray platforms. Analyses of tissues from dorsal and ventral telencephalon from wildtype ( $n=14)$, Ngn1-/-, Ngn2-/-, Mash1-/-, Ngn1-/-; Ngn2-/ -, and Ngn2-/-; Mash1-/- transgenic mice and GOF tissues from mice in which Ngn2 or Mash1 were electroporated on E10.5 and killed $18 \mathrm{~h}$ later (see above) are included in this dataset. Analyses of Ngn1, Ngn2, and Mash1 single and double knockouts were performed with RNA extracted from tissue dissected from E13.5 mice and hybridized to U74A and U74B Affymetrix chips. Basal ganglia from Mash1 KO embryos were dissected and processed for RNA trizol extraction as described previously [4]. Microarray data from cortical tissue have been described previously [4]. Two replicates of each control and a single knockout were analyzed, whereas one replicate for each double knockout and control was sampled. Microarray analyses of dorsal and ventral telencephalic tissues from control and GOF mice (E10.5 mice cultured for $18 \mathrm{~h}$ ) were performed using the Affymetrix MOE430 2.0 chip. Replicates were performed for a total of two dorsal telencephalon controls, three ventral telencephalon controls, and three each of the Ngn2 and Mash1 GOF mice. Normalization was performed using GC-RMA software for background adjustment using sequence information [83] downloaded from [84]. MOE and U74 probesets were assigned Ensembl IDs based on Ensembl Version 37 and duplicate Ensembl IDs were collapsed within a set by taking the median value. Gene expression ratios used for the subsequent network analyses described below were derived from individual mutant arrays versus a timematched wild-type control gene expression array. To gen- 
erate a list of putative target genes we used a 1.3-fold cutoff. We note that the use of a fold change cut-off has been shown to be more reliable than $p$-value or false discovery rate (FDR) cut-offs, in a multicenter large-scale quality control analysis across laboratories and platforms [85]. Furthermore, our target gene lists are generated from two independent fold change cut-offs, from both transgenic and GOF experiments, thereby increasing confidence in the resultant target gene lists. GOF experiments were performed at an earlier stage in neurogenesis (E10.5 and cultured for $18 \mathrm{~h}$ ) than LOF experiments (E13.5) so that expression of Mash1 and Ngn2 in the telencephalon is still low and the effect of overexpressing these genes is maximal, while the LOF analysis had been performed at a stage when Mash1 and Ngn2 expression is high, to maximize the effect of loss of these genes. Based on previous analysis of Mash1 and Ngn2 function in telencephalic development $[3,4]$, we do not expect these genes to have substantially different functions and target genes at these two stages.

\section{Quantification of networks}

The strength of the relationships in GRNs were quantified to calculate the posterior probability distribution for the strength of the linkages based on the fold changes seen in the gene expression datasets [8]. A log-linear function was used to describe relationships between genes:

$$
\ln \left(G_{i}\right)=\alpha_{i}+\sum_{j=1, j \neq i}^{n} I_{j i} \beta_{j i} \ln \left(G_{j}\right)+\varepsilon_{i}
$$

where $\alpha_{I}$ is the level of gene expression independent of the network, $I_{j i}$ is an indicator function $(0,1,-1)$ if a linkage exists from gene $j$ to gene $i, \beta_{j i}$ is the degree to which change in gene $j$ will affect change in gene $i, G_{j}$ is a variable associated with the relative expression level of gene $j$ compared with normal level $j, e_{i}$ is the random error in predicted value for gene $i$ and $n$ is the number of genes in the network.

The posterior distributions for the linkages in each network were derived using Markov chain Monte Carlo (MCMC) sampling methods as described elsewhere $[8,34]$. For the current analysis, KO and GOF effects on genes are modeled as dedicated parents where the prior for $\alpha_{i}$ is set to zero; all other $\alpha$ are assumed to have normal priors. The priors for the $\beta$ are assumed normal with mean zero and variance $\sigma=1$. Finally, $e_{i}$ is assumed to be normally distributed with mean zero and variance $\sigma_{2}$, where $\sigma_{2}$ is assumed to have a uniform prior with support defined by the observed data. The MCMC maximum sampling step sizes are 0.05 for the $\sigma, 0.08$ for the $\beta$, and 0.05 for the $\alpha$, and 500,000 iterations were performed with decimation of every 10 th value. The last 50,000 iterations were used to establish the mean value of $\beta_{j i}$ and the significance of this value. Statistical significance of the parameter $\beta_{j i}$ is defined by less than $5 \%$ of iterations with $\beta_{j i} \leq 0$. To address the specificity of our method, we have permuted the gene labels from the microarray experiments $(n=12,357)$ generating 100 random datasets of gene expression. We then applied these datasets to quantitate the lit-based network to determine the number of times we see significance of these connections from each randomly generated dataset. It should be noted that gene expression correlations across experimental conditions are preserved in this analysis. Software for performing these analyses is available from JMG.

\section{Identification of potential co-factors for Ngn2 and Mash I} Consensus binding sites for Ngn2 and Mash1 were defined as CANTWG and GCAGSTGK, or CAGSTG, respectively, based in part on $[12,28,29]$ and unpublished data (DSC and FG) as described in Additional file 7. Due to the scale of the bioinformatics method performed for predicting co-factors, we limited our analysis to the sequence surrounding 11 predicted Ngn2 target genes, 14 predicted Mash 1 genes, and 6 common target genes based on criterion similar to that used for in situ confirmation in that we focused on the most differentially expressed as well as the best candidate genes from the literature (Additional file 8). For each gene we looked at a minimum of $500 \mathrm{~kb}$ of sequences in front of (approximately $300 \mathrm{~kb}$ ) and behind (approximately $200 \mathrm{~kb}$ ) including UTRs and introns of the gene of interest and surrounding genes that fell within the $500 \mathrm{~kb}$ range. We utilized the ECR browser [86] to align human sequence with Mus musculus, Gallus gallus, Xenopus tropicalis, Fugu rubripes, and Danio rerio [87]. Sometimes no conserved regions were found within our search limits, in which case we removed alignments with the lower vertebrates (F. rubripes and D. rerio) and only analyzed alignments to G. gallus and/or X. tropicalis to find conserved non-coding regions. To further refine the alignment, the web-based Mulan program was utilized, which performs a full local multi-sequence alignment that can account for evolutionary reshuffling and inversions using the threaded blockset aligner program $[87,88]$. From this analysis, evolutionary conserved regions (ECRs) with a minimum length of $100 \mathrm{bp}$ and minimal percentage identity of $70 \%$ were defined. Finally, we applied Multitf, which searches across the identified ECRs for conserved TFBSs [88], to search for putative Mash1 (GCAGSTGK or CAGSTG) and Ngn2 (CANWTG) binding sites. In total, 160 conserved Ngn2 and 75 conserved Mash1 binding sites were identified. These sites were distributed over the $500 \mathrm{~kb}$ analyzed, although the highest number of sites was found in the $20 \mathrm{~kb}$ of sequence surrounding the TSS (Additional file 12). Specifically, 19 Mash1 sites were found surrounding the 11 Ngn2 target genes (average number of sites per gene is 
1.7) versus 56 Mash 1 sites surrounding the 20 Mash 1 and common target genes (average number of sites per gene is $2.8)$. However, this difference is not statistically significant $(p=0.2)$. Furthermore, Ngn2 binding sites are found just as often in front of Ngn2 targets versus Mash1 and/or common target genes ( $82 \mathrm{Ngn} 2$ sites were found surrounding the 14 Mash 1 targets and 22 Ngn2 sites were found surrounding the 6 common targets versus 58 sites found surrounding the $11 \mathrm{Ngn} 2$ target genes). The similarity between Ngn2 and Mash1 and potential function in central nervous system development suggests Mash1 targets could be regulated by Ngn2 as well, in the telencephalon or other tissues. In addition, we note that Mash1 target genes are not equivalent to a random set of genes when analyzing for enrichment of Ngn2 sites. With regards to enrichment of Ebox sites in our putative target genes, the CONFAC analysis described below allowed us to show that several Ebox matrices were significantly enriched in the sequence surrounding our predicted Ngn2 and Mash1 target genes when compared with 250 randomly selected genes (Additional file 9).

To identify potential co-factors, we searched for all vertebrate TRANSFAC annotated TFBSs within 30 bp upstream and downstream of the putative Ngn2 or Mash1 binding sites. The $30 \mathrm{bp}$ length was based in part on prior research showing active modules containing Pou and bHLH binding sites within $15 \mathrm{bp}$ of each other [12]. We removed those TRANSFAC annotated TFBSs that overlapped considerably with the putative Ngn 2 and Mash 1 sites including the following TRANSFAC matrices: E12, E2A, Heb, Hen1, Hand1, E47, Ebox, myogenin, NeuroD, Myod, Areb6, Tal1, Lbp1, Ap4, E47, and lmo2com. We also collapsed all similar TRANSFAC matrices that referenced the same family of transcription factors (for example, Pou domain containing factors and SRY domain containing factors) or were for the same transcription factor, but identified in different vertebrate species. TRANSFAC matrices were mapped to current mouse gene identifiers by following the original reference for the matrix found in TRANSFAC through the literature. To identify the most likely co-factors for Ngn2 and Mash1, we performed the Fisher's exact two-sided test with $p<0.05$ to test for significantly enriched TFBSs in sequence surrounding Ngn2 sites versus Mash1 sites or vice versa. Gene expression from wild-type dorsal and ventral telencephalon tissue was analyzed to predict differential dorsal or ventral expression patterns of the predicted co-factors using a 1.5fold change cut-off. These were subsequently compared with in situ analyses found in online databases (Additional file 10).

\section{Identification of putative co-regulators of Ngn2 and Mash I targets}

Promoter region sequence (10,000 bp upstream of TSS) from mouse and human orthologs of Ngn2 predicted targets and Mash1 and Mash1/Ngn2 predicted common targets (only those with RefSeq IDs associated with them) was automatically uploaded from the UCSC database via the CONFAC website [89]. CONFAC then identifies conserved TFBSs from the TRANSFAC database version 7.0 in the human and mouse sequence alignments [30]. As part of the CONFAC software, the Mann-Whitney statistical test was then applied to test for enrichment of TFBSs in the given gene lists. We compared each list with a list of 250 randomly picked genes available from the CONFAC website, as well as comparing our Ngn2 list with the Mash1/ common targets list and vice versa. We then annotated the resulting lists of enriched TRANSFAC TFBSs as described above. Transcription factors that did not show minimal expression ( $>4.5$ median intensity) in wild-type microarray datasets were not analyzed further.

\section{Algorithm-based network structure}

The TAO-Gen algorithm identifies the optimal gene regulatory network given a specific gene expression dataset [34]. Briefly, our method utilizes a log-linear model (Equation 1) and MCMC to identify the network that best accounts for the variability seen in the microarray datasets. In order to explore larger networks, the number of possible networks in the search space is restricted. This is accomplished through use of an annealing algorithm that combines aspects of the Metropolis algorithm used for MCMC sampling and a simulated annealing algorithm used for optimizations. The maximum number of parents for any given gene is restricted to three; however, no complexity penalty was used. Based on standard techniques in Bayesian networks [90], we include all network structures within the top $95 \%$ of scores based on the maximum likelihood and build a common network that includes those interactions that occur in more than $50 \%$ of these network structures. A detailed description, as well as a complete evaluation of this method through statistical simulation studies has been described previously [34]. We have also performed detailed comparisons to another Bayesian network algorithm [35], which was subsequently coded for Matlab [36]. Results are described in detail in Additional file 1.

An informative prior structure was built utilizing several different data sources. The informative prior structure is represented as a matrix with 0 meaning forbidden connection, 1 meaning required connection, and 0.5 meaning no prior information is available. We considered 25 literature-based connections as required connections, based on previous literature data that is consistent with the current microarray data, which are highlighted in the resulting 
network. Genes whose functions are known and do not include direct transcription factor activity or DNA/RNA binding are forbidden from being parents, with the exception of the signaling molecules Wnt7b and Dll1, which are known initiators of transcription via $\mathrm{Wnt} / \beta$-catenin and Notch pathways, respectively.

Parents are required to be expressed in the same tissue as children (greater than 4.5 median intensity ( $\log 2$ base) in wild-type datasets); therefore, solely dorsally expressed genes are not permitted to parent ventrally expressed genes and vice versa. Each TFBS information source is given an informative prior value of 0.1 , such that if a TFBS is found in a sequence in front of a given gene, the prior score is raised from 0.5 to 0.6. TFBS data were derived from both comparative genomics analyses described above. Results obtained with and without the prior structure are described in Additional file 1.

\section{Authors' contributions}

JMG, OA, GG, CJP, and FG designed the research. OA, CZ, DSC, and LN performed the experimental research. JMG, FMP, MVS, and JSP performed the computational and bioinformatics research. JMG, OA, CJP, and FG wrote the manuscript. All authors read and approved the final manuscript.

\section{Additional material}

\section{Additional file 1}

Description of the literature-based network shown in Figure 1. Comparison of the TAO-Gen algorithm with other Bayesian based algorithms. Click here for file

[http://www.biomedcentral.com/content/supplementary/1741-

7007-6-15-S1.doc]

\section{Additional file 2}

Expression of the Ngn2 target NeuroD analyzed by in situ hybridization in the dorsal telencephalon after electroporation of $\mathrm{Ngn} 2$.

Click here for file

[http://www.biomedcentral.com/content/supplementary/1741-

7007-6-15-S2.pdf]

\section{Additional file 3}

List of predicted targets categorized by GO.

Click here for file

[http://www.biomedcentral.com/content/supplementary/1741-

7007-6-15-S3.pdf]

\section{Additional file 4}

List of targets analyzed using in situ hybridization in wild-type and mutant embryos ( Ngn2-/- and Mash1-/-), and of embryos overexpressing Ngn2 or Mash1.

Click here for file

[http://www.biomedcentral.com/content/supplementary/1741-

7007-6-15-S4.pdf]

\section{Additional file 5}

Quantitative PCR analysis of predicted Mash1 targets in P19 cells following transfection of a Mash1 expression vector.

Click here for file

[http://www.biomedcentral.com/content/supplementary/17417007-6-15-S5.pdf]

\section{Additional file 6}

Quantification of literature-based network structure.

Click here for file

[http://www.biomedcentral.com/content/supplementary/17417007-6-15-S6.doc]

\section{Additional file 7}

Determination of Ngn2 and Mash1 consensus binding sites.

Click here for file

[http://www.biomedcentral.com/content/supplementary/1741-

7007-6-15-S7.pdf]

\section{Additional file 8}

List of Ngn2, Mash1, and common target genes examined in co-factor/coregulator analyses.

Click here for file

[http://www.biomedcentral.com/content/supplementary/1741-

7007-6-15-S8.pdf]

\section{Additional file 9}

CONFAC results predicting co-regulators for Ngn2 targets and common targets and enrichment of Ebox sites surrounding Ngn2 and Mash1 target genes, as well as differential gene expression of predicted co-regulators. Click here for file

[http://www.biomedcentral.com/content/supplementary/17417007-6-15-S9.doc]

\section{Additional file 10}

Corroboration of predicted co-factors, co-regulators and related proteins via interrogation of online databases.

Click here for file

[http://www.biomedcentral.com/content/supplementary/1741-

7007-6-15-S10.doc]

\section{Additional file 11}

Matrix of connectivity of algorithm-based gene regulatory network structure for dorsal and ventral telencephalon development.

Click here for file

[http://www.biomedcentral.com/content/supplementary/17417007-6-15-S11.pdf]

\section{Additional file 12}

Histogram of positions of putative Ngn2 and Mash1 binding sites from TSS.

Click here for file

[http://www.biomedcentral.com/content/supplementary/17417007-6-15-S12.xls]

\section{Acknowledgements}

This work was supported by grants from the European Commission Research and Technological Development Programme and the Human Frontiers Science Programme and by institutional funds from the Medical 
Research Council (UK) to FG. This work was supported in part by the Intramural Research Program of the NIH, National Institute of Environmental Health Sciences. We thank the IGBMC (Illkirch, France) and Kenny Campbell and the Children's Hospital Research Foundation (Cincinnati, USA) for performing the microarray experiments and Thomas Dickmeis for helpful comments on manuscript organization.

\section{References}

I. Bertrand N, Castro DS, Guillemot F: Proneural genes and the specification of neural cell types. Nat Rev Neurosci 2002, 3:517-530.

2. Fode C, Ma QF, Casarosa S, Ang SL, Anderson DJ, Guillemot F: A role for neural determination genes in specifying the dorsoventral identity of telencephalic neurons. Genes Dev 2000, 1 4:67-80.

3. Casarosa S, Fode C, Guillemot F: Mash I regulates neurogenesis in the ventral telencephalon. Development 1999, I 26:525-534.

4. Schuurmans C, Armant O, Nieto M, Stenman JM, Britz O, Klenin N, Brown C, Langevin LM, Seibt J, Tang H, Cunningham JM, Dyck R, Walsh C, Campbell K, Polleux F, Guillemot F: Sequential phases of cortical specification involve Neurogenin-dependent and independent pathways. EMBO J 2004, 23:2892-2902.

5. Basso K, Margolin AA, Stolovitzky G, Klein U, Dalla-Favera R, Califano $A$ : Reverse engineering of regulatory networks in human $B$ cells. Nat Genet 2005, 37:382-390.

6. Segal E, Shapira M, Regev A, Pe'er D, Botstein D, Koller D, Friedman $\mathrm{N}$ : Module networks: identifying regulatory modules and their condition-specific regulators from gene expression data. Nat Genet 2003, 34:166-176.

7. Friedman $\mathrm{N}$ : Inferring cellular networks using probabilistic graphical models. Science 2004, 303:799-805.

8. Toyoshiba H, Yamanaka T, Sone H, Parham FM, Walker NJ, Martinez J, Portier CJ: Gene interaction network suggests dioxin induces a significant linkage between aryl hydrocarbon receptor and retinoic acid receptor beta. Environ Health Perspect 2004, I | 2: 12 17- I 224 .

9. Bernard A, Hartemink AJ: Informative structure priors: joint learning of dynamic regulatory networks from multiple types of data. Pacific Symposion on Biocomputing 2005 (PSB05) 2005:459-470.

10. Grove EA, Fukuchi-Shimogori T: Generating the cerebral cortical area map. Annu Rev Neurosci 2003, 26:355-380.

II. Ross SE, Greenberg ME, Stiles CD: Basic helix-loop-helix factors in cortical development. Neuron 2003, 39:13-25.

12. Castro DS, Skowronska-Krawczyk D, Armant O, Donaldson IJ, Parras C, Hunt C, Critchley JA, Nguyen L, Gossler A, Gottgens B, Matter JM, Guillemot F: Proneural bHLH and Brn proteins coregulate a neurogenic program through cooperative binding to a conserved DNA motif. Dev Cell 2006, I I:83 I-844.

13. Akamatsu W, Fujihara H, Mitsuhashi T, Yano M, Shibata S, Hayakawa Y, Okano HJ, Sakakibara S, Takano H, Takano T, Takahashi T, Noda $\mathrm{T}$, Okano H: The RNA-binding protein HuD regulates neuronal cell identity and maturation. Proc Natl Acad Sci USA 2005, 102:4625-4630.

14. Mattar P, Britz O, Johannes C, Nieto M, Ma L, Rebeyka A, Klenin N Polleux $F$, Guillemot $F$, Schuurmans C: A screen for downstream effectors of Neurogenin2 in the embryonic neocortex. Dev Biol 2004, 273:373-389.

15. Ge WH, He F, Kim KJ, Blanchi B, Coskun V, Nguyen L, Wu XB, Zhao J, Heng JIT, Martinowich K, Tao JF, Wu H, Castro D, Sobeih MM, Corfas G, Gleeson JG, Greenberg ME, Guillemot F, Sun YE: Coupling of cell migration with neurogenesis by proneural bHLH factors. Proc Natl Acad Sci USA 2006, I03: 1319 - 1324.

16. Dufour A, Seibt J, Passante L, Depaepe V, Ciossek T, Frisen J, Kullander K, Flanagan JG, Polleux F, Vanderhaeghen P: Area specificity and topography of thalamocortical projections are controlled by ephrin/Eph genes. Neuron 2003, 39:453-465

17. Theil T: Gli3 is required for the specification and differentiation of preplate neurons. Dev Biol 2005, 286:559-57I.

18. Soma K, Shiomi K, Keino-Masu K, Masu M: Expression of mouse Coiled-coil-DIXI (CcdI), a positive regulator of Wnt signaling, during embryonic development. Gene Expr Patterns 2006, 6:325-330
19. Huang HP, Liu M, El-Hodiri HM, Chu K, Jamrich M, Tsai MJ: Regulation of the pancreatic islet-specific gene BETA2 (neuroD) by neurogenin 3. Mol Cell Biol 2000, 20:3292-3307.

20. Mellitzer G, Bonne S, Luco RF, Van de Casteele M, Lenne-Samuel N, Collombat P, Mansouri A, Lee J, Lan M, Pipeleers D, Nielsen FC, Ferrer J, Gradwohl G, Heimberg H: IAI is NGN3-dependent and essential for differentiation of the endocrine pancreas. $E M B O$ J 2006, 25: I 344-1352.

21. Fode C, Ma Q, Casarosa S, Ang SL, Anderson DJ, Guillemot F: A role for neural determination genes in specifying the dorsoventral identity of telencephalic neurons. Genes Dev 2000, 14:67-80.

22. Stenman J, Toresson H, Campbell K: Identification of two distinct progenitor populations in the lateral ganglionic eminence: implications for striatal and olfactory bulb neurogenesis. Neurosci 2003, 23:167-174.

23. Cheng LP, Arata A, Mizuguchi R, Qian Y, Karunaratne A, Gray PA, Arata S, Shirasawa S, Bouchard M, Luo $P$, Chen CL, Busslinger $M$, Goulding M, Onimaru H, Ma QF: TIx3 and TIxI are post-mitotic selector genes determining glutamatergic over GABAergic cell fates. Nat Neurosci 2004, 7:510-5I7.

24. Kotani M, Tajima Y, Osanai T, Irie A, Iwatsuki K, Kanai-Azuma M, Imada M, Kato H, Shitara H, Kubo H, Sakuraba H: Complementary DNA cloning and characterization of RANDAM-2, a type I membrane molecule specifically expressed on glutamatergic neuronal cells in the mouse cerebrum. J Neurosci Res 2003 , 73:603-6I3

25. Pirot P, van Grunsven LA, Marine JC, Huylebroeck D, Bellefroid E): Direct regulation of the Nrarp gene promoter by the notch signaling pathway. Biochem Biophys Res Commun 2004, 322:526-534.

26. Lamar E, Deblandre G, Wettstein D, Gawantka V, Pollet N, Niehrs C, Kintner C: Nrarp is a novel intracellular component of the Notch signaling pathway. Genes Dev 200I, 15:1885-1899.

27. Ishitani $T$, Matsumoto $K$, Chitnis $A B$, Itoh M: Nrarp functions to modulate neural-crest-cell differentiation by regulating LEFI protein stability. Nat Cell Biol 2005, 7: I I06-I I I 2.

28. Powell LM, zur Lage PI, Prentice DRA, Senthinathan B, Jarman AP: The proneural proteins atonal and scute regulate neural target genes through different E-box binding sites. Mol Cell Biol 2004, 24:9517-9526.

29. Seo S, Lim JW, Yellajoshyula D, Chang LW, Kroll KL: Neurogenin and NeuroD direct transcriptional targets and their regulatory enhancers. EMBO J 2007, 26:5093-5108.

30. Karanam S, Moreno CS: CONFAC: automated application of comparative genomic promoter analysis to DNA microarray datasets. Nucleic Acids Res 2004, 32:W475-W484

31. Magdaleno S, Jensen P, Brumwell CL, Seal A, Lehman K, Asbury A Cheung T, Cornelius T, Batten DM, Eden C, Norland SM, Rice DS, Dosooye N, Shakya S, Mehta P, Curran T: BGEM: an in situ hybridization database of gene expression in the embryonic and adult mouse nervous system. PLoS Biol 2006, 4:497-500.

32. Visel A, Thaller C, Eichele G: GenePaint.org: an atlas of gene expression patterns in the mouse embryo. Nucleic Acids Res 2004, 32:D552-D556.

33. Mishra GR, Suresh M, Kumaran K, Kannabiran N, Suresh S, Bala P, Shivakumar K, Anuradha N, Reddy R, Raghavan TM, Menon S, Hanumanthu G, Gupta M, Upendran S, Gupta S, Mahesh M, Jacob B, Mathew P, Chatteriee P, Arun KS, Sharma S, Chandrika KN, Deshpande N, Palvankar K, Raghavnath R, Krishnakanth R, Karathia H, Rekha B, Nayak R, Vishnupriya G, Kumar HGM, Nagini M, Kumar GSS, Jose R, Deepthi P, Mohan SS, Gandhi TKB, Harsha HC, Deshpande KS, Sarker M, Prasad TSK, Pandey A: Human protein reference database 2006 update. Nucleic Acids Res 2006, 34:D4II-D4|4.

34. Yamanaka T, Toyoshiba H, Sone H, Parham FM, Portier CJ: The TAO-Gen algorithm for identifying gene interaction networks with application to SOS repair in E-coli. Environ Health Perspect 2004, I I 2:1614-1621.

35. Geiger D, Heckerman D: Learning Gaussian networks. Proceedings of the Tenth Conference on Uncertainty in Artifical Intelligence 1994:235-243.

36. Werhli AV, Grzegorczyk M, Husmeier D: Comparative evaluation of reverse engineering gene regulatory networks with relevance networks, graphical gaussian models and bayesian networks. Bioinformatics 2006, 22:2523-253I. 
37. Ohtsuka T, Ishibashi M, Gradwohl G, Nakanishi S, Guillemot F, Kageyama R: Hes I and Hes5 as Notch effectors in mammalian neuronal differentiation. EMBO J 1999, I 8:2196-2207.

38. James $A B$, Conway $A M$, Morris $B$ ): Regulation of the neurona proteasome by Zif268 (Egr I). J Neurosci 2006, 26: I624-I634.

39. Gachon F, Fonjallaz P, Damiola F, Gos P, Kodama T, Zakany Duboule D, Petit B, Tafti M, Schibler U: The loss of circadian PAR bZip transcription factors results in epilepsy. Genes Dev 2004, I8:1397-1412

40. Zhu J, Zhang Y, Joe GJ, Pompetti R, Emerson SG: NF-Ya activates multiple hematopoietic stem cell (HSC) regulatory genes and promotes HSC self-renewal. Proc Natl Acad Sci USA 2005 I02: I I728-II733.

4I. Akiyama H, Kim JE, Nakashima K, Balmes G, Iwai N, Deng JM, Zhang ZP, Martin JF, Behringer RR, Nakamura T, de Crombrugghe $B$ : Osteo-chondroprogenitor cells are derived from Sox 9 expressing precursors. Proc Natl Acad Sci USA 2005 , | 02: |4665-|4670.

42. Shalizi A, Gaudilliere B, Yuan ZQ, Stegmuller J, Shirogane T, Ge QY, Tan Y, Schulman B, Harper JW, Bonni A: A calcium-regulated MEF2 surnoylation switch controls postsynaptic differentiation. Science 2006, 3 I I: 1012-1017

43. Vidal M: Interactome modeling. FEBS Lett 2005, 579: |834-|838.

44. Dearden P, Akam M: Segmentation in silico. Nature 2000, 406: $|3|-\mid 32$.

45. Csete ME, Doyle JC: Reverse engineering of biological complexity. Science 2002, 295: 1664-1669.

46. Kim JK, Gabel HW, Kamath RS, Tewari M, Pasquinelli A, Rual JF, Kennedy S, Dybbs M, Bertin N, Kaplan JM, Vidal M, Ruvkun G: Functional genomic analysis of RNA interference in C-elegans. Science 2005, 308: I I64-I I67.

47. Davidson EH, Rast JP, Oliveri P, Ransick A, Calestani $C$, Yuh $\mathrm{CH}$ Minokawa T, Amore G, Hinman V, Arenas-Mena C, Otim O, Brown CT, Livi CB, Lee PY, Revilla R, Rust AG, Pan ZJ, Schilstra MJ, Clarke PJC, Arnone MI, Rowen L, Cameron RA, McClay DR, Hood L, Bolouri $\mathrm{H}$ : A genomic regulatory network for development. Science 2002, 295:1669-1678.

48. Bemstein BE, Mikkelsen TS, Xie XH, Kamal M, Huebert DJ, Cuff J, Fry B, Meissner A, Wernig M, Plath K, Jaenisch R, Wagschal A, Feil R, Schreiber SL, Lander ES: A bivalent chromatin structure marks key developmental genes in embryonic stem cells. Cell 2006, 125:315-326.

49. Hallikas O, Palin K, Sinjushina N, Rautiainen R, Partanen J, Ukkonen E, Taipale J: Genome-wide prediction of mammalian enhancers based on analysis of transcription-factor binding affinity. Cell 2006, I 24:47-59.

50. Woolfe A, Goodson M, Goode DK, Snell P, McEwen GK, Vavouri T, Smith SF, North P, Callaway H, Kelly K, Walter K, Abnizova I, Gilks W, Edwards YJK, Cooke JE, Elgar G: Highly conserved non-coding sequences are associated with vertebrate development. PLoS Biol 2005, 3: I 16-130

51. Parras CM, Schuurmans C, Scardigli R, Kim J, Anderson DJ, Guillemot F: Divergent functions of the proneural genes Mash I and Ngn2 in the specification of neuronal subtype identity. Genes Dev 2002, 16:324-338.

52. Benjanirut $C$, Paris M, Wang WH, Hong SJ, Kim KS, Hullinger RL, Andrisani OM: The cAMP pathway in combination with BMP2 regulates $P h o x 2 a$ transcription via CAMP response element binding sites. I Biol Chem 2006, 28 I:2969-298I.

53. Saxlund MA, Sadler-Riggleman I, Skinner MK: Role of basic helixloop-helix (bHLH) and CREB transcription factors in the regulation of Sertoli cell androgen-binding protein expression. Mol Reprod Dev 2004, 68:269-278.

54. Mandolesi G, Gargano S, Pennuto M, Illi B, Molfetta R, Soucek L, Mosca L, Levi A, Jucker R, Nasi S: NGF-dependent and tissuespecific transcription of vgf is regulated by a CREB-p300 and bHLH factor interaction. FEBS Lett 2002, 5 I 0:50-56.

55. Uittenbogaard M, Martinka DL, Johnson PF, Vinson C, Chiaramello A: 5 ' UTR of the neurogenic bHLH NexI/MARH-2/NeuroD6 gene is regulated by two distinct promoters through CRE and C/EBP binding sites. J Neurosci Res 2007, 85: I- 18.

56. Kurisaki K, Kurisaki A, Valcourt U, Terentiev AA, Pardali K, ten Dijke $P$, Heldin CH, Ericsson J, Moustakas A: Nuclear factor YYI inhibits transforming growth factor beta- and bone morphogenetic protein-induced cell differentiation. Mol Cell Biol 2003, 23:4494-4510
57. Yeh TS, Lin YM, Hsieh RH, Tseng MJ: Association of transcription factor YYI with the high molecular weight notch complex suppresses the transactivation activity of notch. J Biol Chem 2003, 278:41963-4I969.

58. Sugimori M, Nagao M, Bertrand N, Parras CM, Guillemot F, Nakafuku M: Combinatorial actions of patterning and HLH transcription factors in the spatiotemporal control of neurogenesis and gliogenesis in the developing spinal cord. Development 2007, 134:1617-1629.

59. Parras CM, Hunt C, Sugimori M, Nakafuku M, Rowitch D, Guillemot F: The proneural gene Mash I specifies an early population of telencephalic oligodendrocytes. I Neurosci 2007, 27:4233-4242.

60. Kuhlbrodt K, Herbarth B, Sock E, Enderich J, Hermans-Borgmeyer I, Wegner M: Cooperative function of POU proteins and SOX proteins in glial cells. J Biol Chem I998, 273: I6050-I6057.

6I. Zwilling S, Konig H, Wirth T: High-mobility group protein-2 functionally interacts with the Pou domains of octamer transcription factors. EMBO | | 995, | 4: | | 98- | 208.

62. Phillips K, Luisi B: The virtuoso of versatility: POU proteins that flex to fit. I Mol Biol 2000, 302:1023-1039.

63. Vaccarino FM, Schwartz ML, Raballo R, Nilsen J, Rhee J, Zhou M, Doetschman T, Coffin JD, Wyland JJ, Hung YTE: Changes in cerebral cortex size are governed by fibroblast growth factor during embryogenesis. Nat Neurosci 1999, 2:246-253.

64. Bagheri-Fam S, Barrionuevo F, Dohrmann U, Gunther T, Schule R, Kemler R, Mallo M, Kanzler B, Scherer G: Long-range upstream and downstream enhancers control distinct subsets of the complex spatiotemporal Sox9 expression pattern. Dev Biol 2006, 29 I:382-397.

65. Gunhaga L, Marklund M, Sjodal M, Hsieh JC, Jessell TM, Edlund T: Specification of dorsal telencephalic character by sequential Wnt and FGF signaling. Nat Neurosci 2003, 6:70I-707.

66. Backman M, Machon O, Mygland L, van den Bout CJ, Zhong WM, Taketo MM, Krauss S: Effects of canonical Wnt signaling on dorso-ventral specification of the mouse telencephalon. Dev Biol 2005, 279: 155-168.

67. Abu-Khalil A, Fu L, Grove EA, Zecevic N, Geschwind DH: Wnt genes define distinct boundaries in the developing human brain: implications for human forebrain patterning. J Comp Neurol 2004, 474:276-288.

68. Teo JL, Ma H, Nguyen C, Lam C, Kahn M: Specific inhibition of CBP/beta-catenin interaction rescues defects in neuronal differentiation caused by a presenilin-I mutation. Proc Nat Acad Sci USA 2005, 102:12171-12176.

69. Bolognani F, Tanner DC, Merhege M, Deschenes-Furry J, Jasmin B, Perrone-Bizzozero NI: In vivo post-transcriptional regulation of GAP-43 mRNA by overexpression of the RNA-binding protein HuD. I Neurochem 2006, 96:790-80I.

70. Rida PCG, Le Minh N, Jiang YJ: A Notch feeling of somite segmentation and beyond. Dev Biol 2004, 265:2-22.

7I. Kim S, Imoto S, Miyano S: Dynamic Bayesian network and nonparametric regression for nonlinear modeling of gene networks from time series gene expression data. Biosystems 2004, 75:57-65

72. Langdon T, Hayward P, Brennan K, Wirtz-Peitz F, Sanders P, Zecchini $V$, Friday A, Balayo $T$, Arias AM: Notch receptor encodes two structurally separable functions in Drosophila: a genetic analysis. Dev Dyn 2006, 235:998-1013.

73. Katoh M, Katoh M: Notch ligand, JAG I, is evolutionarily conserved target of canonical WNT signaling pathway in progenitor cells. Int J Mol Med 2006, I 7:68I-685.

74. Satoh W, Gotoh T, Tsunematsu Y, Aizawa S, Shimono A: Sfrp I and Sfrp2 regulate anteroposterior axis elongation and somite segmentation during mouse embryogenesis. Development 2006, I33:989-999.

75. Yeang CH, Mak HC, McCuine S, Workman C, Jaakkola T, Ideker T: Validation and refinement of gene-regulatory pathways on a network of physical interactions. Genome Biol 2005, 6(7):R62.

76. Qin $Q Y$, Inatome R, Hotta A, Kojima M, Yamamura $H$, Hirai $H$, Yoshizawa T, Tanaka H, Fukami K, Yanagi S: A novel GTPase, CRAG, mediates promyelocytic leukemia protein - associated nuclear body formation and degradation of expanded polyglutamine protein. I Cell Biol 2006, I 72:497-504.

77. Osumi N, Inoue T: Gene transfer into cultured mammalian embryos by electroporation. Methods 200I, 24:35-42. 
78. Niwa H, Yamamura K, Miyazaki J: Efficient selection for highexpression transfectants with a novel eukaryotic vector. Gene 1991, 108:193-199.

79. Beckers J, Caron A, de Angelis MH, Hans S, Campos-Ortega JA, Gossler A: Distinct regulatory elements direct Delta I expression in the nervous system and paraxial mesoderm of transgenic mice. Mech Dev 2000, 95:23-34.

80. Ikuta T, Yoshida N, Satoh N, Saiga H: Ciona intestinalis Hox gene cluster: Its dispersed structure and residual colinear expression in development. Proc Natl Acad Sci USA 2004, 101:15118-15123.

8I. Farah MH, Olson JM, Sucic HB, Hume RI, Tapscott SJ, Turner DL: Generation of neurons by transient expression of neura bHLH proteins in mammalian cells. Development 2000, 1 27:693-702.

82. Johnson JE, Birren SJ, Anderson DJ: 2 Rat homologs of DrosophilaAchaete-Scute specifically expressed in neuronal precursors. Nature 1990, 346:858-86I.

83. Irizarry RA, Hobbs B, Collin F, Beazer-Barclay YD, Antonellis KJ, Scherf U, Speed TP: Exploration, normalization, and summaries of high density oligonucleotide array probe level data. Biostatistics 2003, 4:249-264.

84. Bioconductor 2005 [http://www.bioconductor.org]

85. Guo L, Lobenhofer EK, Wang C, Shippy R, Harris SC, Zhang L, Mei $N$, Chen T, Herman D, Goodsaid FM, Hurban P, Phillips KL, Xu J, Deng XT, Sun YMA, Tong WD, Dragan YP, Shi LM: Rat toxicogenomic study reveals analytical consistency across microarray platforms. Nat Biotechnol 2006, 24: I |62-I I69.

86. ECR browser [http://ecrbrowser.dcode.org/]

87. Loots GG, Ovcharenko I: Dcode.org anthology of comparative genomic tools. Nucleic Acids Res 2005, 33:W56-W64.

88. Ovcharenko I, Loots GG, Giardine BM, Hou MM, Ma J, Hardison RC Stubbs L, Miller W: Mulan: Multiple-sequence local alignment and visualization for studying function and evolution. Genome Res 2005, 15: 184-194.

89. CONFAC website: UCSC database. [http://morenolab.white head.emory.edu/cgi-bin/confac/login.pl].

90. Chickering DM: A transformational characterization of equivalent Bayesian network structures. Proceedings of the Twelfth Annual Conference on Uncertainty in Artificial Intelligence (UAI) 1996:87-98.
Publish with Bio Med Central and every scientist can read your work free of charge

"BioMed Central will be the most significant development for disseminating the results of biomedical research in our lifetime. "

Sir Paul Nurse, Cancer Research UK

Your research papers will be:

- available free of charge to the entire biomedical community

- peer reviewed and published immediately upon acceptance

- cited in PubMed and archived on PubMed Central

- yours - you keep the copyright
BioMedcentral 